\title{
Crystallographic Aspects of the Propanoate Salts
}

\section{Jan Fábry, Erika Samolová}

\author{
Institute of Physics of the Czech Academy of Sciences, Praha 8, Czech Republic;
}

$$
\text { fabry@fzu.cz }
$$

The propionic acid is the first simple carboxylic acid in the series of simple carboxylic acids starting from the formate where the hydrophobic character prevails. The propanoates make a text-book example of structures in which hydrophilic/cation-oxygen interactions and hydrophobic interactions (between the ethyl groups) are combined resulting in a rich variety of structures.

The structural motifs thus result in layer-like strutures, columnar structures or isolated clusters in which the inner part consists of a structural part where the hydrophilic interactions prevail in contrast to an outer part where the hydrophobic interaction dominate.

The structures are affected by the positional disorder of the ethyl chains.

Water inclusion into the structures is quite frequent which stabilizes the hydrophilic interactions in the structures.

\section{Keywords: propanoates, carboxylate groups}

This work was supported by the Czech Science Foundation (Project No. 19-28594X). Dr. Ivana Císařová from the Faculty of Science of the Charles University in Prague is thanked for the measurement of some samples 